\title{
Die „Friedensdividende“ der Globalisierung: Außenwirtschaftliche Öffnung und innenpolitische Stabilität in den Entwicklungsländern
}

\author{
Margit Bussmann / Harald Scheuthle / Gerald Schneider
}

Eine der umstrittenen Folgewirkungen der globalen wirtschaftlichen Integration besteht im möglichen Effekt, den die außenwirtschaftliche Öffnung auf den gesellschaftlichen Zusammenhalt ausübt. Während der freihändlerische Liberalismus eine Stabilisierung erwartet, sind die Globalisierungskritiker deutlich skeptischer. Sie gehen zumindest in der kurzen Frist von einer Destabilisierung aus. In diesem Aufsatz untersuchen wir diese widerstreitenden Interpretationen für die Entwicklungsländer. In dieser Staatengruppe war der „Sturmlauf zum Freihandel“, wie Rodrik den Paradigmenwandel in der Außenwirtschaftspolitik beschrieb, besonders ausgeprägt. Unsere Resultate widerlegen für eine Vielzahl von Indikatoren der politischen Stabilität die Befürchtung, die Hinwendung zum Weltmarkt unterminiere die oft fragilen Entwicklungsländer. Einen signifikant positiven Effekt übt die außenwirtschaftliche Öffnung aber nur bedingt aus.

\section{Einleitung ${ }^{l}$}

Als im Frühjahr 2002 Zehntausende von Argentiniern gegen die Wirtschaftspolitik ihres Landes protestierten, richtete sich der Zorn nicht zuletzt gegen die in den 1990er Jahren betriebene außenwirtschaftliche Liberalisierung. So hatte der lateinamerikanische Staat wie seine Nachbarn nach Jahrzehnten der Abschottung den Empfehlungen des IWF Folge geleistet und seine Kapital- und Gütermärkte systematisch geöffnet. Globalisierungskritiker sahen sich durch die argentinische Wirtschaftskrise in ihrer Skepsis bestätigt, die sie den Deregulierungs- und Öffnungsrezepten des „Washingtoner Konsensus" von Beginn an entgegen gebracht hatten. Nach dieser Interpretation verschärft die wirtschaftliche Integration die sozialpolitischen Konflikte, da besonders die Kapitalbesitzer und Exporteure von der Abkehr von der Importsubstitution und anderen protektionistischen Rezepten profitierten, die Arbeiterschaft und die heimische Industrie aber unter der neuen Politik verlören.

In diesem Sinne wäre zu erwarten, dass der „Rush to Free Trade“ (Rodrik 1994), den viele Entwicklungsländer in den letzten zwei Jahrzehnten erfasst hat, von einer zu-

1 Der Aufsatz beruht auf der Konstanzer Diplomarbeit des zweiten Autors und ist eine erste Analyse im Rahmen eines größeren Forschungsprojektes. Margit Bussmann und Gerald Schneider möchten sich bei der Deutschen Stiftung Friedensforschung für die finanzielle Unterstützung und bei Nina Wiesehomeier, Indra de Soysa sowie zwei PVS-Gutachtern für Kommentare bedanken. Die Daten, die in der Analyse Verwendung fanden, sind auf der Replikationshomepage des dritten Autors verfügbar: http://www.uni-konstanz.de/FuF/Verwiss/ GSchneider/downloads/daten.htm. 
nehmenden gesellschaftlichen und politischen Instabilität begleitet ist. Doch obwohl diese These in der Globalisierungsdebatte eine zentrale Rolle spielt, fehlt es bis jetzt an systematischen Tests. So beschäftigt sich der größte Teil der einschlägigen Literatur mit den Ursachen und nicht etwa den Folgen der ökonomischen Interdependenz. Auch die Bürgerkriegsforschung, die in den letzten Jahren eine zunehmende Professionalisierung erfahren hat, verweist nur zum Teil auf ökonomische Bedingungen von innerstaatlichen Konflikten. Besonderes Gewicht kommt dabei dem Rohstoffbesitz zu (Collier 2001, de Soysa 2002); die Verflechtung eines Staates mit der Weltwirtschaft spielt in den quantitativ-empirischen Analysen aber höchstens die Rolle einer Kontrollvariablen. Dies ist ganz im Gegensatz zu den 1970er und 1980er Jahren, als Vertreter der Dependenzansätze auf die angeblich destabilisierende Wirkung einer Integration in die Weltwirtschaft verwiesen. Frühere Kolonialstaaten oder Länder, die wie Kolonien in die Weltwirtschaft integriert seien, erführen „unvollständige Wirtschaftskreisläufe, eine krasse soziale Zerklüftung und eine aus beiden Faktoren zwangsweise resultierende politische Instabilität", argumentierte etwa Senghaas (1988: 164-165). Auch wenn Weede (1990) und andere diese Dependenz-Thesen zurückwiesen, tauchte die Befürchtung in jüngerer Zeit wieder in der deutschsprachigen Literatur auf (Schlichte 1996, Kurtenbach/Mehler 2002).

Zwar beschäftigen sich quantitativ orientierte Studien schon seit langem mit den ökonomischen Ursachen von Protestverhalten (z.B. Jagodzinski 1983). Systematische Untersuchungen, inwieweit außenwirtschaftliche Öffnung mit politischer Instabilität zusammenhängt, liegen bis jetzt jedoch nicht vor. Dabei hat Rogowski (1989) das Problem schon vor über zehn Jahren aufgeworfen. Er zeigte aufgrund gängiger handelstheoretischer Modelle auf, wie sich Freihandel oder Protektion auf Klassengegensätze und andere Konfliktlinien auswirken. Auch andere Autoren sehen eine Handelsliberalisierung im Zusammenhang mit einem Regimewechsel oder mit wirtschaftlichen Krisen (Fernandez/Rodrik 1991: 1147), während Alesina und Drazen (1991) die Verzögerung notwendiger Reformen auf den Zermürbungskampf zwischen konkurrierenden Interessen zurückführen.

Nach Auffassung der Globalisierungskritiker (z.B. Adams u.a. 1999, Hoogvelt 2000) geht ein Reformland das Risiko ein, über den Öffnungskurs den Grad an politischer Instabilität markant zu erhöhen. Grundsätzlich kann außenwirtschaftliche Öffnung den Zusammenhalt einer Gesellschaft kurz- oder langfristig beeinflussen. In diesem Aufsatz untersuchen wir diese beiden Effekte separat. So ist etwa denkbar, dass eine Gesellschaft zwar kurz vor und nach dem Vollzug von außenwirtschaftlichen Liberalisierungsmaßnahmen von sozialpolitischen Unruhen geschüttelt ist, der durch die Öffnung herbeigeführte Wachstumseffekt langfristig diese Konflikte aber entschärft. Um die Wirkungsweisen von Öffnungsmaßnahmen genauer zu erfassen, unterscheiden wir auch zwischen verschiedenen Formen von Instabilität, nämlich gewaltfreien Massenprotesten, politischer Gewalt und Staatsversagen.

Unser Artikel ist folgendermaßen aufgebaut: Zunächst stellen wir aufgrund der Außenwirtschaftstheorie dar, unter welchen Bedingungen und in welchem Zeitraum Öffnungsmaßnahmen auf innenpolitischen Widerstand stoßen können. Wir leiten dazu unsere Leithypothesen zu den kurz- und langfristigen Effekten der Öffnung ab und kontrastieren diese Erklärungen mit Kontrollhypothesen, die wir der neueren Literatur 
zu den Ursachen von Bürgerkriegen entnommen haben. In den Ausführungen zum Forschungsdesign operationalisieren wir die wesentlichen Konstrukte und stellen unser Schätzmodell näher vor. Wir präsentieren die Ergebnisse in bivariater und multivariater Form, wobei auch für das zentrale Modell konditionale Effekte berechnet werden. Der Artikel schließt mit der Zusammenfassung und einem Ausblick auf die nächsten Forschungsschritte, die wir im Anschluss an diese Globalanalyse zu beschreiten trachten.

\section{Außenwirtschaftliche Offenheit und innenpolitische Stabilität: Theorie und Hypothesen}

Nur eine verschwindende Minderheit von Ökonomen bezweifelt heute, dass Handel gesamtwirtschaftlich positive Folgen für ein Land hat und die Wohlfahrt steigert (Frey et al. 1984). In der neoklassischen Perspektive führt Handel zu einer effizienteren Arbeitsteilung zwischen den Ländern. Freihandelsorientierte Staaten nutzen demnach ihre komparativen Kostenvorteile und können so mehr produzieren und konsumieren als Autarkien. Die Preise für Güter nähern sich angesichts der internationalen Konkurrenz dem Weltmarktpreis, der unterhalb des durch protektionistische Maßnahmen verzerrten nationalen Preises liegt. Sachs und Warner (1995) weisen nach, dass offene Entwicklungsländer höhere Wachstumsraten haben und leichter Zahlungsbilanzkrisen verhindern können als Staaten, die einen protektionistischen Kurs verfolgen. Plümper (2001) qualifiziert dieses Argument, bestreitet aber nicht, dass der Abbau von Handelshemmnissen positive Wirkungen hat. Um diese Wohlfahrtsgewinne ausnutzen zu können, müssen in seiner Sicht die regulativen Bedingungen für einen florierenden Freihandel gegeben sein.

Eine freihändlerische Orientierung kann das Risiko von innenpolitischem Konflikt auf zwei verschiedene Arten vermindern. Erstens vergrößert Handel den Wohlstand der breiten Bevölkerung und reduziert somit Faktoren, die nach der Deprivationstheorie (Gurr 1970) und ähnlichen Ansätzen zu Instabilität führen können. Nach dem Stolper-Samuelson-Theorem, einem zentralen Baustein der Außenhandelstheorie, profitiert von diesem Wohlstand jeweils der Faktor, der in einem Land reichhaltig vorhanden ist. In den Entwicklungsländern ist das, je nachdem, wie man die Faktoren aufteilt, entweder Arbeit oder unausgebildete Arbeit (Wood 1994). In jedem Fall ist es die breite Masse der Bevölkerung, die von der Öffnung profitiert. Es stellt sich jedoch die Frage, ob außenwirtschaftliche Öffnung auch zu mehr Einkommensgleichheit führt. Hier ist die empirische Evidenz zwiespältig. Nach Fischer (2001) ist die Einkommensungleichheit nach der Öffnung in Südostasien gesunken, während sie in Lateinamerika gestiegen ist. Andere Studien finden entweder keinen Zusammenhang oder kommen zu dem Schluss, dass Öffnung zu einer gerechteren Verteilung führt (zum Beispiel Bourguignon/Morrisson 1990; Edwards 1997; Bussmann u.a. 2002). Wenn Freihandel tatsächlich zu mehr Einkommensgleichheit führt, dann kann das zusätzlich die Gefahr von politischen Konflikten senken, und zwar wieder über die Abschwächung jener Faktoren, die politischen Protest hervorrufen. Zufriedene Bürger protestieren nach der Deprivationstheorie weniger gegen den Staat als arme und benachteiligte. 
Zweitens führen die Gewinne der außenwirtschaftlichen Öffnung dazu, dass das Interesse wächst, den Handel aufrechtzuerhalten. Jeder gewaltsame Konflikt verhindert dies jedoch. Deshalb liegt es im Interesse von Staaten und deren Bürgern, innenpolitischen Frieden und Stabilität zu gewährleisten. Dieser zweite Argumentationsstrang, der für die wirtschaftliche Öffnung spricht, ist eine Weiterentwicklung des liberalen oder demokratischen Friedens. Im Rückgriff auf Kant behaupten verschiedene Forscher, dass demokratische und in den Welthandel eingebundene Staaten einem reduzierten Risiko ausgesetzt sind, gegeneinander zu den Waffen zu greifen. Da Krieg die Handelsströme zwischen den Staaten abbreche, seien Waffengänge zu kostspielig für einen Staat und dessen Bürger (Russett/Oneal 2001). Außerdem verstärke der Handel liberale Normen, die gewaltsamen Formen des Konfliktaustrags zwischen Demokratien ächten (Kant 1795 [1987]). Dieses Argument, angewandt auf zwischenstaatliche Kriege, ist schon vielfach theoretisch diskutiert und mehrheitlich positiv empirisch überprüft worden (siehe z.B. Barbieri/Schneider 1999; Russett/Oneal 2001; Schneider, Barbieri und Gleditsch 2003). Dieselbe Logik lässt sich auch auf innerstaatliche Konflikte anwenden. Extreme Formen der innenpolitischen Instabilität können demnach den freien Handel behindern und deshalb potenzielle Gewinne reduzieren, die eine Gesellschaft auf lange Sicht durch einen Kurs der außenwirtschaftlichen Öffnung erzielen könnte. ${ }^{2}$ Die relevanten Akteure werden also Freihandel in ihr Kosten-Nutzen-Kalkül einbeziehen, wenn sie ihre innenpolitischen Strategien überdenken. Eine Eskalation der Auseinandersetzungen um die außenwirtschaftliche Orientierung ist deshalb für alle Beteiligten kontraproduktiv, weil solche Streitigkeiten ausländische Investoren und Handelspartner abschrecken. Wenn es also um die Schädlichkeit eines eskalierenden innenpolitischen Konflikts geht, sitzen alle, Gewerkschaften und Arbeitgeber, diskriminierte und protegierte Industrien, in einem Boot.

Bezüglich der langfristigen Folgen von außenwirtschaftlicher Öffnung gehen wir also davon aus, dass die Offenheit den Staaten zu einer Steigerung der wirtschaftlichen Entwicklung verhilft. Dadurch vermindert sich der Anreiz für die wirtschaftspolitischen Kontrahenten, ihre Konflikte gewaltsam zu lösen. Zusätzlich werden durch den Handel liberale Normen der friedlichen Konfliktbewältigung verbreitet, wie der Transaktionalismus seit einem halben Jahrhundert postuliert (Deutsch u.a. 1957). Aufgrund dieser verschiedenen Wirkungsstränge vermuten wir, dass offenere Staaten weniger anfällig für politische Instabilität sind. Wir fassen diese Überlegungen in einer ersten $\mathrm{Hy}$ pothese zusammen.

H1: Je offener ein Staat ist, desto geringer ist sein Risiko für politische Instabilität.

Während auf lange Sicht die wohlfahrtssteigernde Wirkung von außenwirtschaftlicher Offenheit entscheidend scheint, ist auf kurze Sicht vor allem die Umverteilungswirkung des Öffnungsprozesses wichtig. Wenn sich eine geschlossene Volkswirtschaft öffnet, ist das ein schwerwiegender Eingriff in bestehende Wirtschafts- und Sozialbeziehungen. Vor allem Entwicklungsländer, die in großem Maße auf Importsubstitution

2 Andererseits können Rebellen und manchmal auch Regierungen von der Kriegswirtschaft profitieren, die sich in manchen Staaten etablieren, die massiv unter politischer Gewalt leiden. Dies trifft besonders auf Staaten zu, die reich an Rohstoffen sind (Collier 2000). 
und verwandte Strategien gesetzt haben, müssen bei einer Handelsöffnung mit Einkommenseinbußen bei einem großen Teil der Bevölkerung rechnen.

Die Behauptung, dass der außenwirtschaftliche Öffnungsprozess das Instabilitätsrisiko erhöhen kann, lässt sich mit beiden klassischen Ansätzen der Außenhandelstheorie verbinden, dem Ricardo-Viner- wie auch dem bereits erwähnten Stolper-SamuelsonAnsatz, dessen Hauptthesen heute vor allem im Heckscher-Ohlin-Modell Verwendung finden (Hiscox 2001). Auf die zweite Denktradition stützt sich die viel beachtete Arbeit von Rogowski (1989). Seiner Meinung nach aktiviert eine Änderung des Handelsregimes die Koalitionsmuster dermaßen, dass die betroffene Gesellschaft instabiler wird. Er unterscheidet aufgrund der Vorarbeiten von Stolper und Samuelson die Faktoren ,Kapital', ,Arbeit' und ,Land'. Danach profitieren bei einer Wendung zum Freihandel die Halter der reichlich vorhandenen Faktoren, während die Halter der knappen Faktoren verlieren. Während sich die Verlierer gegen diese Veränderung stemmen, streben die Gewinner nach einer Beschleunigung, um ihre politische Macht auszubauen (Rogowski 1989: 5). Von dieser Dynamik ausgehend, leitet Rogowski für den Übergang vom Protektionismus zum Freihandel Konfliktlinien ab, die vom Verhältnis zwischen Land, Kapital und Arbeit gekennzeichnet sind: Stadt gegen Land in Staaten, in denen Land der reichlich vorhandene Faktor ist, und Arbeit gegen Kapital in Staaten, in denen der reichliche Faktor die Arbeit ist.

Einen ähnlichen Ansatz liefert Frieden (1991). Er argumentiert aufgrund des Ricardo-Viner-Modells, das von sektorspezifischen Faktoren ausgeht. Dies steht im Gegensatz zum Ansatz von Stolper und Samuelson sowie dem Heckscher-Ohlin-Modell, in denen die Faktoren vollständig mobil zwischen den Sektoren sind. Aufgrund der unterschiedlichen Annahmen verläuft im sektorspezifischen Modell die Konfliktlinie also nicht zwischen den unterschiedlichen Klassen, sondern zwischen dem exportorientierten und dem importkonkurrierenden Sektor. In der Logik des Ansatzes von Frieden (1991) lässt sich vermuten, dass der Einfluss eines Sektors von der Spezifität der eingesetzten Faktoren und der Kohäsion der betroffenen Industrien abhängt.

Diese theoretischen Überlegungen greifen auch für die Entwicklungsländer, deren politische Ökonomie bis zum „Wettlauf nach Freihandel“ (Rodrik 1994) weitgehend durch eine staatlich regulierte Wirtschaft und durch Rent-Seeking geprägt war. Beides führte nicht unbedingt zum Wohlstand des Landes, sondern eher zur Bereicherung der Eliten (Tullock 1980, Weede 1990). Die Regierung verfolgte mit ihrer Wirtschaftspolitik das Ziel, durch Exporte von Ressourcen und landwirtschaftlichen Produkten Devisen zu erwirtschaften, die zum Aufbau von importsubstituierenden Industrien verwendet wurden. Diese Industrien wurden meist von Gefolgsleuten der Regierung unwirtschaftlich geführt und waren darum international nicht wettbewerbsfähig. Um den importkonkurrierenden Sektor zu schützen, erließen opportunistische Regierungen eine Vielzahl von Maßnahmen. So konnten die herrschenden Eliten Renten erwirtschaften und der Regierung als Gegenleistung ihre Loyalität und Unterstützung versprechen (Bates 1981). Dieses System war deshalb mittelfristig stabil, weil weder die Regierung noch die Eliten einen Anreiz hatten, etwas daran zu ändern. Die Folge waren jedoch so hohe volkswirtschaftliche Kosten, dass für viele Länder Strukturreformen und damit auch die außenwirtschaftliche Öffnung mit der Zeit unausweichlich schienen. 
Andere Renten erwachsen in diesen Staaten aufgrund von Bodenschätzen. So erhalten Investoren die Ausbeutungsrechte als Gegenleistung für die finanzielle Unterstützung, die sie der "Staatsklasse“ angedeihen lassen (Clapham 1996:70). Auch solche monopolartigen Verflechtungen zwischen der Regierung und einem Wirtschaftssektor sind auf die Dauer volkswirtschaftlich eine Last, da sie die Etablierung von ineffizienten Austauschverhältnissen fördern. Im Zuge der Verwerfungen, welche das Rentseeking fast unausweichlich erzeugt, ertönt dann der Ruf nach außenwirtschaftlicher Öffnung. Die so erzeugten Krisen sind zwar triviale, aber dennoch bedeutsame Bedingungen für makroökonomische Reformen (Rodrik 1994).

Die Liberalisierung ist aber insofern problematisch, als sie zumindest in der kurzen Frist Verlierer schafft. Rodrik (1994) schätzt, dass das politische Kosten-Nutzen-Verhältnis größer als fünf ist; das heißt, dass für jeden Euro zusätzliches Einkommen durch Öffnung fünf Euro umverteilt werden. Diese immensen Verteilungswirkungen gestalte die Reform politisch überaus schwierig, besonders dann, wenn die Kompensation der Verlierer unzureichend sei. Nach der Logik des Ricardo-Viner-Modells wirkt sich eine Änderung in der Handelspolitik direkt auf das Einkommen des importkonkurrierenden Sektors aus. Die Allgemeinheit dagegen profitiert von den niedrigeren Preisen und den Produktivitätsfortschritten, welche die Reform begleiten, während der Exportsektor über den Produktionszuwachs sein Einkommen steigern kann. Da nicht zwangsläufig mit einer Kompensation zugunsten der Verlierer der Reform zu rechnen ist, spricht sich der Sektor, der unter Importkonkurrenz steht, in dieser Perspektive gegen eine Außenhandelsöffnung aus.

Außenwirtschaftliche Öffnung ist also ein schwieriges Unterfangen, insbesondere wenn die Gefolgsleute der Regierung darunter leiden. Dies ist gerade in den Entwicklungsländern oft der Fall, da sich die staatlichen Haushalte und damit auch die Einkommen der Beamtenschaft wesentlich aus Zöllen und staatlichen Lizenzen alimentieren. Für die Kreise, die bis zur Öffnung vom Protektionismus profitierten, sind die Kosten der Reform nicht kurzfristiger Art. So ist es dem bis dahin protegierten Sektor per definitionem nicht möglich, nach dem Abschluss der Reformphase wieder die üblichen Renten abzuschöpfen. Durch die Handelsliberalisierung versiegt diese Quelle praktisch vollständig. Der neo-patrimonialen Regierung fällt es immer schwerer, ihre Vasallen zu kompensieren, da sie ja selbst auch zunehmend Einnahmequellen verliert. Die Regierungen können sich zwar vielleicht über die Liberalisierung neue Geldquellen erschließen, die sie durchaus auch zum eigenen Nutzen abschöpfen (Bueno de Mesquita u.a. 2000). Solche Motive identifiziert etwa Pawelka (1997: 224) für jene Eliten im Vorderen Orient, die sich für eine politische wie wirtschaftliche Öffnung ihres Landes einsetzen. So sei es bei den Reformen der 1970er Jahre darum gegangen, „entwicklungspolitische Krisen und Legitimationsprobleme dadurch zu überwinden, dass günstige Zugangsbedingungen zu den regionalen und weltweiten Rentenströmen geschaffen wurden“. Doch solche Bereicherungsmotive können nicht verbergen, dass der angestrebte Umbau der Wirtschaftsstruktur zu einer substanziellen Umverteilung führt, die auch bestimmte Teile der Regierung negativ berührt (Plümper und Schneider 2000). Deshalb wäre eigentlich mit Abwehrreaktion des bisher gehätschelten Wirtschaftssektors und dessen Verbündeten im Regierungsapparat zu rechnen. 
Während des außenwirtschaftlichen Öffnungsprozesses sollte der Staat deshalb anfällig für politische Instabilität sein. Auf der einen Seite muss die Regierung die wirtschaftliche Lage verbessern, um Unruhen in der Bevölkerung zu verhindern und der Opposition den Wind aus den Segeln zu nehmen. Auf der anderen Seite muss sie befürchten, dass die bis dahin protegierten Eliten einen Putsch organisieren, um Reformen zu verhindern oder zurückzunehmen. Diese widersprüchlichen Ziele konstituieren jene Zwangslage, die Plümper und Schneider (2000) als das „Trilemma“ des protektionistischen Autokraten bezeichnen. Demnach will der Autokrat einerseits sein eigenes Einkommen und das seiner Vasallen maximieren. Da er jedoch die Stabilität seines Regimes erhalten will, muss er in einer Krisensituation entweder Zugeständnisse an die freihändlerische Opposition oder an die Bevölkerung machen. Problematisch ist dies für den Autokraten deshalb, weil er nur zwei dieser drei Ziele gleichzeitig erreichen kann. Vor diesem Hintergrund ist offensichtlich, weshalb ein lang andauernder sozialpolitischer Konflikt oft die rechtzeitige Umsetzung von Krisenpaketen verhindert (Alesina/Drazen 1991).

Zusätzlich zur ersten Hypothese zum Zusammenhang zwischen Offenheit und politischer Stabilität untersuchen wir deshalb kurzfristige Auswirkungen. Wir vermuten, dass eine Öffnung der Wirtschaft unmittelbar das Risiko für einen innenpolitischen Konflikt erhöht, da dies zu einer weitreichenden Umverteilung im Staat führt, welche die wirtschaftlichen und politischen Gewinner und Verlierer polarisieren kann. Dies gilt besonders deshalb, weil eine massive Umstrukturierung das ganze Land in das sprichwörtliche „Tal der Tränen“ zwängt. Gerade während dieser Transformation erscheinen gleichzeitig die Wohlfahrtsgewinne als äußerst ungewiss, welche die Reformer versprochen haben.

H2: Der Prozess der außenwirtschaftlichen Öffnung führt kurzfristig zu einem erhöhten Instabilitätsrisiko.

Während unser Augenmerk besonders dem Zusammenhang zwischen außenwirtschaftlicher Öffnung bzw. Offenheit und politischer Gewalt gilt, werden wir zusätzlich den Einfluss von weiteren Faktoren erfassen, die in Verbindung mit Instabilität gebracht wurden. In der komparativen Forschung existieren vor allem zwei Ansichten darüber, wie politische Gewalt entsteht. Die eine Theorie rückt wirtschaftliche Unzufriedenheit in den Vordergrund, während die andere eher die günstige Gelegenheit als zentral erachtet (Schock 1996). Die Vertreter der Deprivationstheorie gehen davon aus, dass der von der Bevölkerung wahrgenommene Unterschied zwischen ihren Erwartungen und den tatsächlichen ökonomischen Verhältnissen zu Gewalt führen kann (Gurr 1970). Ungleichheiten heizen auch die Spannungen an, die zwischen unterschiedlichen Bevölkerungsgruppen ohnehin bereits bestehen mögen. Wenn Unzufriedenheit also auf ausgeprägte Gruppenidentitäten trifft, vergrößert sich in dieser Perspektive das Potenzial zur politischen Gewalt (Ellingsen 2000).

Für die Vertreter der Theorie der politischen Opportunität spielen weniger die wirtschaftlichen Bedingungen eine Rolle, sondern vielmehr der politische Kontext und die Möglichkeit, Probleme des kollektiven Handelns auf diese Weise zu überwinden. Faktoren, die ein Potenzial für Gewalt in sich bergen, sind in jeder Gesellschaft vorhanden, aber nur in bestimmten Situationen entlädt sich die potenzielle Gewalt tat- 
sächlich (Tilly 1978; Tarrow 1989, 1998). Dabei spielen vor allem die Charakteristika des politischen Systems eine Rolle. Identität und Frustration sind in dieser Sicht nicht ausreichend, um eine Rebellion auszulösen. Die Gruppe braucht zusätzlich eine günstige Gelegenheit, wobei ihre Führer kühl kalkulieren, ob ein Aufstand Aussicht auf Erfolg hat (Tilly 1978).

Bei der Entstehung von Gewalt spielen also wirtschaftliche, politische und soziokulturelle Faktoren eine Rolle. Die quantitative Literatur nimmt im Gefolge der Modernisierungstheorie an, dass ein hoher Entwicklungsstand die Instabilität senkt, weil die Bevölkerung der Regierung das wirtschaftliche Wohlergehen des Landes zugute hält (Henderson/Singer 2000). Lipset (1959) hat klassisch zu zeigen versucht, dass der wirtschaftliche Wohlstand zu Errungenschaften wie Ausbildung oder zu einem besseren Zugang zu Informationen führt, was in seiner Sicht wiederum die politische Instabilität vermindert. ${ }^{3}$ Weiter sieht Gurr (1979) im wirtschaftlichen Entwicklungsstand ein Mittel, um politische Gewalt zu verringern, ohne dass dabei gleichzeitig politischer Protest vermindert werde, da reiche Länder vor allem Demokratien seien und Demokratien gewaltfreien Protest eher tolerierten. Eine mikrofundierte Version dieses Erklärungsmusters bieten Collier und Hoeffler (1998). Ihnen zufolge steigen die Kosten für eine Rebellion bei höherem Entwicklungsstand, weil jedes einzelne Mitglied einer Rebellion mehr zu verlieren hat. Wir gehen also davon aus, dass wirtschaftlicher Wohlstand die Attraktivität jener Bedingungen mindert, welche die Bevölkerung zu einer Rebellion veranlassen könnten.

H3: Wirtschaftlicher Wohlstand senkt das Risiko der politischen Instabilität.

Eine wirtschaftliche Krise schwächt die Regierung in vielerlei Hinsicht und macht sie somit anfälliger für Rebellionen. Durch geringere Steuereinnahmen wird die Fähigkeit der Regierung eingeschränkt, Renten an verschiedene Gruppen im Staat zu verteilen (Plümper/Schneider 2000). Zweitens führt eine Wirtschaftskrise zu größerer Unzufriedenheit, weil die Bevölkerung die Hauptlast der Krise zu tragen hat und die Regierung dafür verantwortlich macht. Während in einer Demokratie die Regierung damit rechnen muss, bei schlechter wirtschaftlicher Lage abgewählt zu werden, kann es in einer Diktatur zu politischer Gewalt kommen, wenn die Regierung ihre Unterstützer nicht mehr mit Renten versorgen kann, so dass diese deshalb erwägen, die Regierung mit Gewalt abzusetzen. Laut Collier und Hoeffler (2000) erhöht eine wirtschaftliche Krise das Risiko eines innenpolitischen Konfliktes, aber nicht, weil eine Gruppe der Bevölkerung sich dadurch um ihre Renten betrogen fühlt. Eine schlechte wirtschaftliche Lage senkt eher die Rekrutierungskosten für die Rebellen, da sie ihren potenziellen Mitgliedern ein höheres Einkommen versprechen können als die Einkünfte, die sie auf regulären Arbeitsmärkten erreichen würden.

H4: Wirtschaftliches Wachstum senkt das Risiko der politischen Instabilität.

Der Liberalismus als Theorieströmung innerhalb der Internationalen Beziehungen postuliert, dass Demokratien besonders resistent gegenüber gewaltsamen, politischen Kon-

3 Vertreter der These, der Modernisierungsprozesses verlaufe konfliktiv, sehen hingegen im Gegensatz zwischen weiterhin vorhandenen traditionellen und neuen kapitalistischen Vergesellschaftungsformen ein wichtiges Konfliktpotenzial (Schlichte 1996). 
flikten sind. Dafür verantwortlich sind vor allem zwei Mechanismen: kompetitive demokratische Wahlen und das mit ihnen verbundene Institutionengefüge auf der einen und gewaltfreie Normen der Konfliktregelung auf der anderen Seite. In demokratischen Systemen ist daher ein friedlicher Regierungswechsel möglich. Wenn die Mehrheit der Bevölkerung mit der politischen Führung unzufrieden ist, kann sie die Regierung abwählen. Demokratische Normen erhöhen das Potenzial zur friedlichen Konfliktbewältigung und zum gewaltfreien Umgang in Streitfragen (zum Beispiel Russett 1993; Gleditsch 1995; Rummel 1997). Empirisch hat sich weitgehend bestätigt, dass Demokratien die weniger konfliktträchtigen politischen Systeme sind. Allerdings kann hier nicht von einer linearen Verbindung ausgegangen werden, sondern der Zusammenhang zwischen Demokratiegrad und innenpolitischer Gewalt ist umgekehrt u-förmig (Boswell/Dixon 1993; Schock 1996; Auvinen 1997; Ellingsen 2000; Henderson/ Singer 2000; Hegre u.a. 2001). Danach ist das Risiko für politische Instabilität in Autokratien und Demokratien eher gering, während es in Halbdiktaturen hoch ist. Im Unterschied zu demokratischen Politikern sind Autokraten nicht auf die Unterstützung des Volkes angewiesen, sondern werden im Allgemeinen von einer sehr kleinen Schicht getragen. Im Austausch für die Unterstützung ihrer Herrschaft erwartet diese Gruppe, dass sie von der Regierung Renten erhält (Frey/Eichenberger 1994; Pritzl/Schneider 1997). Durch die zahlenmäßig kleine "Gewinnkoalition“ wird das Überleben des autokratischen Herrschers mittelfristig relativ unabhängig von der Leistungsfähigkeit der Wirtschaft (Bueno de Mesquita u.a. 2000), doch in der langen Frist droht es auch ihnen, für ein schlechtes Management bestraft zu werden. Zusätzlich haben Autokratien die Möglichkeit, Widerstand gewaltsam zu unterdrücken.

H5: Das Instabilitätsrisiko ist in Staaten, die nur halbwegs demokratisch organisierten sind, größer als in Autokratien oder Demokratien.

Die Bevölkerungsgröße ist eine weitere Kontrollvariable, der man im Zusammenhang mit außenwirtschaftlicher Öffnung Beachtung schenken sollte. Große Staaten sind nach Alesina et al. (1997) anfälliger für einen innenpolitischen Konflikt als kleine. Aufgrund der zunehmenden Heterogenität der Gesellschaft steigt mit der Bevölkerungsgröße die Wahrscheinlichkeit, dass einzelne Gruppen sich einen Vorteil von einer Sezession versprechen. Dieser Zusammenhang zeigt sich auch in der empirischen Literatur (z.B. Collier/Hoeffler 1998, 2000; Zanger 2000). Zusätzlich zu dem direkten Wirkungspfad, der von der Größe eines Landes auf das Konfliktrisiko hinzeigt, gibt es auch einen indirekten Effekt, der die außenwirtschaftliche Öffnung mit einbezieht. Große Länder sind meist weniger stark in die Weltwirtschaft integriert, da sie die Konsumbedürfnisse ihrer Bevölkerung eher im eigenen Binnenmarkt befriedigen können als kleine Staaten (Alesina/Wacziarg 1998). Dieser Vorteil kommt jedoch zum Preis der größeren kulturellen Heterogenität, der sich in einer verminderten Leistungsfähigkeit der politischen Institutionen ausdrückt. Daraus lässt sich das Argument ableiten, dass in kleineren Ländern eine außenwirtschaftliche Öffnung eher begrüßt wird, da ein größerer Teil der Bevölkerung direkt davon profitiert. In größeren Ländern, die unter Umständen dazu noch heterogen sind, sind die Vorteile der Öffnung relativ gering. In diesen Staaten kann die außenwirtschaftliche Öffnung somit das Risiko für politische Instabilität erhöhen. 
H6: Bevölkerungsreiche Länder haben ein höheres Instabilitätsrisiko als bevölkerungsarme.

\section{Forschungsdesign}

Wir überprüfen die Hypothesen eines möglichen Zusammenhangs zwischen politischer Instabilität und außenwirtschaftlicher Offenheit und Öffnung anhand von Daten für 90 Entwicklungsländer für den Zeitraum von 1978 bis 1997. Wir verwenden als Schätzmethode den Generalized Estimation Equation-Ansatz (GEE). Dabei wählen wir die Option der logistischen Schätzung, da es sich bei den berücksichtigten Instabilitätsformen um dichotome abhängige Variablen handelt. ${ }^{4}$ Eine logistische Regression eruiert aufgrund der unabhängigen Variablen die Wahrscheinlichkeit, dass ein Ereignis eintritt und die abhängige Variable den Wert 1 statt 0 annimmt. In unserem Fall bedeutet dies, dass wir für die untersuchten Staatenjahre die Wahrscheinlichkeit schätzen, dass es zu Instabilität kommt. Im Folgenden beschreiben wir die Operationalisierung der einzelnen Variablen und die Datenquellen.

Abhängige Variable: Politische Instabilität

Wir verwenden drei verschiedene Indikatoren für Instabilität: Massenproteste, politische Gewalt und Staatsversagen. Die Operationalisierung der ersten beiden Indikatoren erfolgt in enger Anlehnung an Jagodzinskis (1983) Empfehlung, zwei Arten von internen Konflikten zu unterscheiden, nämlich kollektive Proteste und politische Gewalt. Für die Variable Massenproteste verwenden wir ebenso wie Jagodzinski Daten zu gewaltsamen Unruhen, Generalstreiks und Antiregierungs-Demonstrationen. Die entsprechenden Angaben haben wir aus der Cross-National Time-Series Database (CNTS Archive 2001) übernommen. Unruhen sind gewaltsame Demonstrationen. Generalstreiks und Antiregierungs-Demonstrationen sind im Gegensatz dazu friedlich, sie richten sich gegen die Regierungspolitik. Außerdem gibt es im CNTS-Datensatz Angaben über revolutionäre und Guerilla-Aufstände und politische Morde, die wir in der Variablen politische Gewalt zusammenfassen. Revolutionen sind alle erfolgreichen oder erfolglosen Rebellionen, die das Ziel verfolgen, die Regierung zu stürzen. Beim Guerilla-Krieg werden die Aktivitäten illegaler Rebellengruppen gezählt. Das Konzept politische Morde umfasst Tötungen oder Tötungsversuche an hohen Regierungsbeamten oder Politikern.

4 Der GEE-Ansatz schätzt ein Durchschnittspopulationsmodell. Demnach wird überprüft, ob ein durchschnittlich geöffnetes oder ein durchschnittlich geschlossenes Land anfälliger für Instabilität ist. Der Vorteil einer Schätzung durch GEE ist, dass wir sämtliche Tests mit den notwendigen Korrekturen für Unabhängigkeitsverletzungen der Regressionsannahmen durchführen können, d.h. wir verwenden White-korrigierte Standardfehler, um dem Problem der Heteroskedastizität entgegenzuwirken. Somit kann die Voraussetzung aufgehoben werden, dass die einzelnen Beobachtungen, zumindest für den einzelnen Untersuchungsstaat, unabhängig sein müssen. Da wir außerdem davon ausgehen müssen, dass in Paneldatensätzen Datenpunkte zeitlich korreliert sind und somit die Unabhängigkeitsannahme weiterhin verletzt wird, kontrollieren wir für Autokorrelation des ersten Grades (Zorn 2001). 
Alle Variablen aus der CNTS-Datenbank zählen die Anzahl der Ereignisse pro Jahr, die von Jagodzinski lediglich addiert und logarithmisch transformiert wurden. Das Zusammenrechnen von verschiedenen Ereignissen, das einem solchen additiven Index zugrunde liegt, erachten wir jedoch als problematisch, insbesondere da in diesem Fall der ordinale Charakter der Variablen mehr als fraglich ist. Stattdessen erstellen wir Indikatoren, die den Wert eins annehmen, wenn ein Instabilitätsereignis auftritt und null wenn nicht. Genauer gesagt untersuchen wir das Konstrukt ,Protest', das den Wert eins hat, wenn es in einem Land in einem Jahr mindestens eine Demonstration, einen Generalstreik und/oder eine Revolte gab. Fand keines dieser Ereignisse statt, hat die Variable den Wert null. Analog sind wir mit der Variablen politische Gewalt und den CNTS-Daten zu revolutionären Aufständen, Guerilla-Aufständen und politischen Morden verfahren. Durch die Benutzung der Boolschen Logik lassen sich also die Erhebungsfehler reduzieren, die bereits Jagodzinski (1983) angesprochen hat.

Zusätzlich zu Massenprotesten und politischer Gewalt untersuchen wir Staatsversagen. Dazu verwenden wir die Daten der State Failure Task Force, die Bürgerkriege, Genozide, Regimewechsel und Putsche umfassen (Esty u.a. 1998). Der von uns verwendete Datensatz beruht auf King und Zeng (2001). Das State Failure Projekt unterteilt die Variable ,Staatsversagen' in revolutionäre Bürgerkriege, ethnische Bürgerkriege, Genozide und Staatskollaps. Um als Bürgerkrieg klassifiziert zu werden, muss ein Konflikt zwischen dem Staat und einer Rebellengruppe ausbrechen, bei dem die Rebellen mindestens 1000 Mitglieder mobilisieren und dem mindestens 100 Tote pro Jahr zum Opfer fallen. Dies unterscheidet sich von der bekannten Definition des Correlates of War-Projekts, bei dem ein Bürgerkrieg mindesten 1000 Tote pro Jahr fordern muss. Revolutionäre Kriege unterscheiden sich von „ethnischen“ durch die unterschiedlichen Ziele der Kombattanten Bei der ersten Form von bewaffneten Konflikten steht im Vordergrund, die Regierung zu stürzen, während bei ethnisch motivierten Bürgerkriegen die aufständische Gruppe versucht, ihren eigenen Status zu verändern. Genozide sind staatliche organisierte Massenmorde, die über mindestens sechs Monate andauern. Staatszusammenbrüche sind abrupte, gewaltsame Änderungen in der Staatsstruktur oder Wechsel hin zu einem autokratischen Regime. Sie sind definiert durch eine Änderung auf der Polity-Skala um mehr als drei Punkte oder durch eine längere Periode, in der stabile staatliche Institutionen fehlen. Wie schon zuvor operationalisieren wir Staatsversagen ebenfalls als $0-1$ Indikator. ${ }^{5}$

Durch die Verwendung von verschiedenen Indikatoren für Instabilität möchten wir gewährleisten, dass unsere Ergebnisse auch bei wechselnden Definition der abhängigen Variable robust bleiben, zumal es in der Forschung noch keinen Konsens gibt, wie politische Instabilität zu messen sei (Auvinen 1997; Boswell/Dixon 1993). ${ }^{6}$

5 Tests mit Bürgerkriegsdaten aus einem neuen Datensatz (Gleditsch u.a. 2002) als abhängige Variable führen zu ähnlichen Ergebnissen (Bussmann/Schneider 2003).

6 Wir haben auf den Einbezug von anderen Formen der politischen Gewalt wie extralegale Tötungen verzichtet, weil dazu keine verlässlichen vergleichenden Datensätze vorliegen. Menschenrechtsverletzungen finden als Indikator keine Berücksichtigung, weil diese Form der Gewaltanwendung eher in Zusammenhang mit Repression als mit Rebellion und Instabilität zu sehen ist. 
Unabhängige Variablen

Außenwirtschaftliche Öffnung und Offenheit: Wir unterscheiden zwischen außenwirtschaftlicher Offenheit, also dem Ausmaß, zu dem ein Staat tatsächlich in die Weltwirtschaft eingebunden ist, und außenwirtschaftlicher Öffnung, also dem Prozess, während dem eine Regierung die regulativen Voraussetzungen für Freihandel schafft. Um die langfristigen Auswirkungen von außenwirtschaftlicher Offenheit zu messen, stützen wir uns auf den Außenhandelsquotienten, der aus der Summe der Exporte und Importe, geteilt durch das BIP, errechnet wird. Dies sorgt dafür, dass für die Landesgröße kontrolliert wird, da kleine Länder generell mehr Handel treiben als große. Die Daten stammen aus den Penn World Tables Version 6.0. Das Problem dieser Messung ist, dass die Handelsströme nicht unbedingt etwas über die Handelspolitik aussagen (Martin u.a. 2001: 3).

,Außenwirtschaftliche Öffnung' bezeichnet die jährliche Veränderung der Bestimmungen, die Regierungen zur Regulierung des Handelverkehrs einsetzen. Der Indikator misst demnach die Abwesenheit von Zöllen, Quoten, Ein- und Ausfuhrbeschränkungen und das Vorhandensein einer frei konvertierbaren Währung. Öffnung bedeutet die Zurücknahme regulativer Hemmnisse, die den Freihandel behindern. Damit ein Land die Vorteile des Freihandels ausnutzen kann, muss es also politische Vorbedingungen erfüllen. Um die kurzfristigen Auswirkungen des Öffnungsprozesses zu untersuchen, verwenden wir deshalb den CACAO-Indikator von Martin, Plümper und Schneider (2001). Dieser Offenheitsindikator misst die Handelsinstitutionen und -beschränkungen auf einer Ordinalskala von 0 für ,offen' bis 7 für ,geschlossen'. Der Index basiert auf einer Kombination von Handelspolitiken und institutionellen Arrangements, die der IWF-Publikation ,Report on Exchange Arrangement and Exchange Restrictions' entnommen wurden. Der Öffnungsprozess wird durch die Differenz der CACAO-Werte zum Vorjahr gemessen, wodurch wir den zeitlichen Charakter des Öffnungsprozesses miteinbeziehen. Durch diesen Indikator ist auch eine Unterscheidung möglich, ob sich ein Land gradualistisch oder radikal liberalisiert hat.

Entwicklungsstand: Empirisch wurde der negative Zusammenhang zwischen dem Entwicklungsstand und der politischen Instabilität oder einem Bürgerkrieg schon häufig nachgewiesen. Das BIP pro Kopf ist praktisch eine Standardvariable in jeder Regression (z.B. Auvinen 1997; Collier/Hoeffler 1998, 2000; Fearon/Laitin 2000; Henderson/Singer 2000; Zanger 2000). Andere Autoren kommen mit der Verwendung des Energieverbrauchs pro Kopf zu ähnlichen Ergebnissen (z.B. Jagodzinski 1983; Ellingsen 2000). Wir entnehmen die Variable BIP pro Kopf den Penn World Tables 6.0 und fügen sie unserem Model in logarithmierter Form bei, um die schiefe Verteilung der Variable auszugleichen.

Wirtschaftswachstum: Empirisch getestet wird der Einfluss von Wirtschaftkrisen nur in wenigen Studien. In Jagodzinskis (1983) Studie hat Wirtschaftswachstum einen negativen bzw. keinen Effekt auf Instabilität. Auvinen (1997) findet einen positiven Zusammenhang zwischen Inflation und politischem Konflikt und einen negativen für das Pro-Kopf-Wirtschaftswachstum. Basierend auf dem BIP pro Kopf wird Wachstum gemessen als $\left(\mathrm{BIP}_{\mathrm{t}}-\mathrm{BIP}_{\mathrm{t}-1}\right) / \mathrm{BIP}_{\mathrm{t}-1}$. 
Demokratiegrad: Wir operationalisieren die Art des Regimes mit dem weitverbreiteten Polity IV-Index (Marshall/Jaggers 2000). Polity kombiniert verschiedene institutionelle Charakteristika des politischen Systems - Offenheit und Wettbewerb bei der Rekrutierung der Exekutive, Machtbeschränkungen der Exekutive und Regulierung und Wettbewerb der politischen Partizipation - zu einem Index von -10 für Diktaturen bis +10 für Demokratien. Wir fügen den Demokratieindex unserem Model für politische Gewalt in einfacher sowie in quadrierter Form bei, um die umgekehrt u-förmige Kurve zu untersuchen.

Bevölkerungsgröße: Für die Operationalisierung dieser Variablen benutzen wir die einschlägigen Angaben der Weltbank (World Bank 1999). Aufgrund der sehr schiefen Verteilung der Messwerte verwenden wir den natürlichen Logarithmus.

Für die Variablen Entwicklungsstand, Wirtschaftswachstum, Demokratiegrad sowie den Handelsvariablen Offenheit und Öffnung benutzen wir die Werte vom Vorjahr, um zu vermeiden, dass diese Variablen von Instabilität beeinflusst wurden. Bei Bevölkerung erschien uns dies aufgrund der relativen Stabilität dieser Variablen nicht notwendig. Tabelle 1 fasst unser Grundmodell mit den zu erwartenden Vorzeichen der unabhängigen Variablen zusammen.

Tabelle 1: Erwarteter Einfluss der unabhängigen Variablen auf politische Instabilität

\begin{tabular}{lc}
\hline Unabhängige Variable & Erwarteter Einfluss auf politische Instabilität \\
\hline Offenheit & - \\
Öffnung & + \\
Wirtschaftswachstum & - \\
Entwicklungsstand & - \\
Demokratiegrad & + \\
Quadrierter Demokratiegrad & - \\
Bevölkerung & + \\
\hline
\end{tabular}

\section{Ergebnisse}

Wir werfen einen ersten Blick auf die Frage, ob offene Staaten stabiler sind als geschlossene und betrachten die Verteilung der Offenheit und die der Instabilität in einer Kreuztabelle. Offenheit ist dabei in drei Kategorien aufgeteilt: von geschlossen (0 bis 2 auf der CACAO-Offenheitsskala) über eine Mittelkategorie (3 bis 4) zu offen (5 bis 7). Als Instabilität sehen wir uns kollektive Proteste, politische Gewalt und Staatsversagen an. Die Ergebnisse werden in Form von Balkendiagrammen präsentiert (Abbildungen 1-3).

Nur für politische Gewalt und Staatsversagen können wir durch ein signifikantes $\chi^{2}$ Unterschiede zwischen den Gruppen finden, das heißt, dass offene Länder weniger unter Instabilität leiden. Während in den geschlossenen und mittleren Kategorien von Offenheit das Verhältnis zwischen Nicht-Gewalt und Gewalt ca. 2:1 beträgt, ist das Verhältnis bei den außenwirtschaftlich offenen Staaten etwa 9:1. Im Fall von Protesten können diese Unterschiede rein zufällig sein. Hier können wir erkennen, dass die Ver- 
Abbildung 1: Handelsoffenheit und Massenprotest

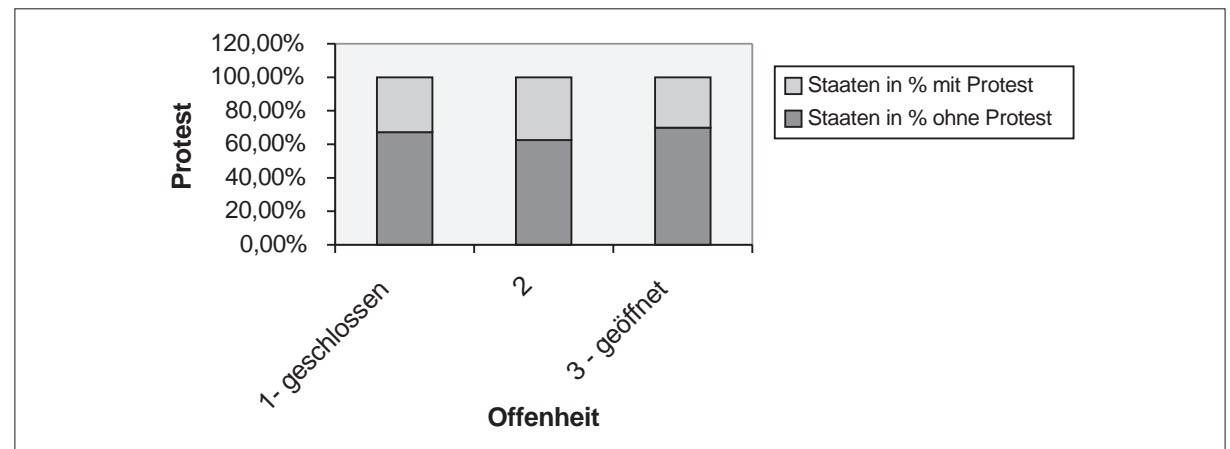

Abbildung 2: Handelsoffenheit und politische Gewalt

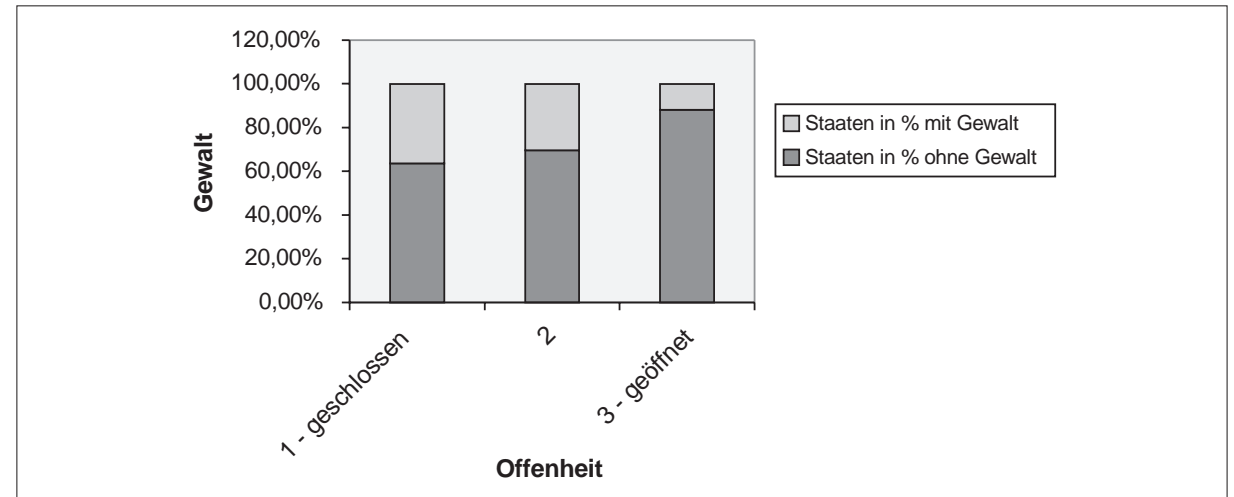

Abbildung 3: Handelsoffenheit und Staatsversagen

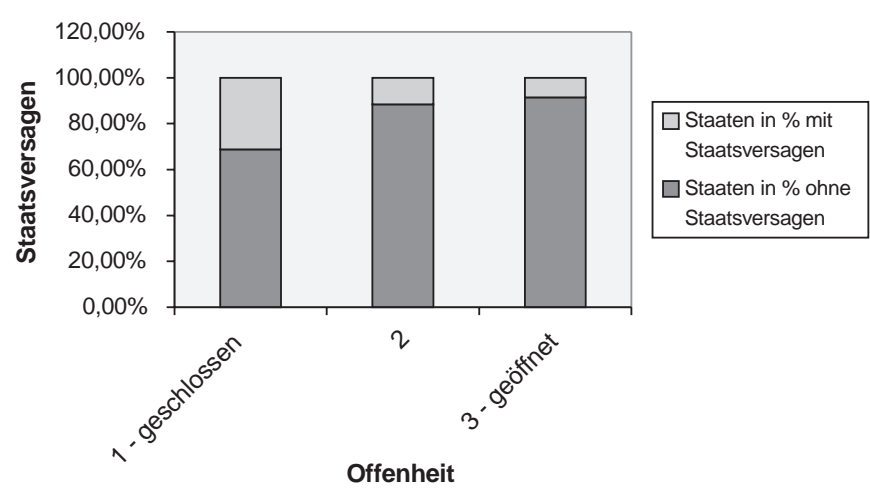


teilung über alle drei Kategorien von Öffnung hinweg 2:1 beträgt. Eine rein bivariate Betrachtungsweise bringt uns zu dem Schluss, dass Offenheit zwar in keinem Zusammenhang mit friedlichem Protestverhalten einer Bevölkerung steht, jedoch mit politischer Gewalt systematisch zu variieren scheint.

Unsere eigentlichen Tests bestehen jedoch aus multivariaten Regressionen, bei denen wir den Zusammenhang zwischen Offenheit und Instabilität untersuchen und gleichzeitig zusätzliche Faktoren konstant halten. Die erste Gruppe von Modellen konzentriert sich auf verschiedene Indikatoren von politischer Instabilität; die Resultate dazu sind in Tabelle $2 \mathrm{zu}$ finden.

Tabelle 2: Außenwirtschaftliche Offenheit und Öffnung und verschiedene Indikatoren innenpolitischer Instabilität, 1978 - 1997

\begin{tabular}{|c|c|c|c|c|c|c|}
\hline & \multicolumn{2}{|c|}{ Massenprotest } & \multicolumn{2}{|c|}{ Politische Gewalt } & \multicolumn{2}{|c|}{ Staatszusammenbruch } \\
\hline & Modell 1 & Modell 2 & Modell 3 & Modell 4 & Modell 5 & Modell 6 \\
\hline Offenheit $t-1$ & $\begin{array}{c}-0,0085 \\
(0,0049)\end{array}$ & - & $\begin{array}{c}-0,0090^{*} \\
(0,0046)\end{array}$ & - & $\begin{array}{c}-0,0006 \\
(0,0047)\end{array}$ & - \\
\hline Öffnung $\mathrm{t}_{-1}$ & - & $\begin{array}{c}-0,0088 \\
(0,113)\end{array}$ & - & $\begin{array}{c}-0,061 \\
(0,144)\end{array}$ & - & $\begin{array}{c}-0,048 \\
(0,077)\end{array}$ \\
\hline $\begin{array}{l}\text { Entwicklungs- } \\
\text { stand }_{t-1}\end{array}$ & $\begin{array}{l}0,514^{* *} \\
(0,157)\end{array}$ & $\begin{array}{c}0,397^{*} \\
(0,165)\end{array}$ & $\begin{array}{c}0,014 \\
(0,159)\end{array}$ & $\begin{array}{c}-0,035 \\
(0,181)\end{array}$ & $\begin{array}{c}-0,361 \\
(0,253)\end{array}$ & $\begin{array}{c}-0,055 \\
(0,282)\end{array}$ \\
\hline $\begin{array}{l}\text { Wirtschafts- } \\
\text { wachstum }{ }_{t-1}\end{array}$ & $\begin{array}{l}-1,74 \\
(0,99)\end{array}$ & $\begin{array}{l}-3,36^{* *} \\
(1,21)\end{array}$ & $\begin{array}{c}-0,345 \\
(0,769)\end{array}$ & $\begin{array}{c}-0,702 \\
(1,146)\end{array}$ & $\begin{array}{c}0,297 \\
(0,418)\end{array}$ & $\begin{array}{c}0,436 \\
(0,474)\end{array}$ \\
\hline $\begin{array}{l}\text { Demokratie- } \\
\operatorname{grad}_{t-1}\end{array}$ & $\begin{array}{l}0,049^{* * *} \\
(0,014)\end{array}$ & $\begin{array}{c}0,043^{* *} \\
(0,016)\end{array}$ & $\begin{array}{l}0,045^{* *} \\
(0,016)\end{array}$ & $\begin{array}{l}0,059^{* * *} \\
(0,018)\end{array}$ & $\begin{array}{c}0,062^{*} \\
(0,026)\end{array}$ & $\begin{array}{c}0,052 \\
(0,028)\end{array}$ \\
\hline $\begin{array}{l}\text { Quadrierter De- } \\
\text { mokratiegrad }_{\mathrm{t}-1}\end{array}$ & $\begin{array}{l}-0,0014 \\
(0,0030)\end{array}$ & $\begin{array}{c}-0,0001 \\
(0,0037)\end{array}$ & $\begin{array}{c}-0,0079^{*} \\
(0,0039)\end{array}$ & $\begin{array}{c}-0,0069 \\
(0,0044)\end{array}$ & $\begin{array}{c}-0,0018 \\
(0,0040)\end{array}$ & $\begin{array}{r}-0,0006 \\
0,0049\end{array}$ \\
\hline Bevölkerung & $\begin{array}{l}0,41^{* * *} \\
(0,09)\end{array}$ & $\begin{array}{l}0,51^{* * *} \\
(0,09)\end{array}$ & $\begin{array}{c}0,13 \\
(0,11)\end{array}$ & $\begin{array}{c}0,20^{*} \\
(0,09)\end{array}$ & $\begin{array}{l}0,53^{* * *} \\
(0,13)\end{array}$ & $\begin{array}{l}0,51^{\text {*** }} \\
(0,14)\end{array}$ \\
\hline Konstante & $\begin{array}{c}-10,76^{* * *} \\
(1,92)\end{array}$ & $\begin{array}{c}-12,16^{* * *} \\
(1,65)\end{array}$ & $\begin{array}{c}-1,73 \\
(2,13)\end{array}$ & $\begin{array}{c}-3,34 \\
(2,13)\end{array}$ & $\begin{array}{c}-6,92^{*} \\
(2,79)\end{array}$ & $\begin{array}{c}-8,95^{* *} \\
(3,10)\end{array}$ \\
\hline $\mathrm{N}$ & 1442 & 1050 & 1345 & 986 & 1445 & 1050 \\
\hline Wald $\mathrm{Chi}^{2}$ & 76,93 & 69,42 & 26,25 & 25,91 & 23,88 & 20,18 \\
\hline Prob > Chi ${ }^{2}$ & 0,0001 & 0,0001 & 0,0002 & 0,0002 & 0,0006 & 0,003 \\
\hline
\end{tabular}

Anmerkung: Die Zahlen stellen Koeffizienten dar, in Klammern sind die semi-robusten Standardfehler ausgewiesen. Die Sterne stellen das Signifikanzniveau dar $\left(^{*} \mathrm{P}<0,05\right.$; ${ }^{* *} \mathrm{P}<0,01$; *** $\mathrm{P}<0,001)$.

Das erste Modell zeigt die langfristigen Auswirkungen von Offenheit auf Massenprotest, also das Auftreten von Demonstrationen, Streiks und/oder Aufruhr. Offenheit ist negativ mit Massenprotesten verbunden, verfehlt jedoch knapp das .05 Signifikanzniveau $(\mathrm{p}<.08)$. Die Nullhypothese einer fehlenden Beziehung zwischen Offenheit und Stabilität kann also nicht eindeutig verworfen werden. In Modell 2 ersetzen wir dann die langfristig angelegte Offenheit mit der Variable Öffnung für die kurzfristigen Auswirkungen des Öffnungsprozesses. Auch hier lässt sich ein negativer Zusammenhang be- 
obachten, allerdings mit hoher Fehlerwahrscheinlichkeit. Kurzfristige Auswirkungen von außenwirtschaftlicher Öffnung auf das Protestverhalten in Entwicklungsländern konnten also in unseren ersten Tests nicht entdeckt werden.

Die Kontrollvariablen haben in beiden Modellen die erwarteten Vorzeichen. Die Ergebnisse von Entwicklungsstand lassen vermuten, dass die Hypothese, reichere Länder würden weniger unter Konflikten leiden als arme, nicht berechtigt ist, zumindest nicht bezüglich des Protestverhaltens. Stattdessen lässt sich in reichen Ländern mehr Protestverhalten beobachten, ein Ergebnis, das statistisch signifikant ist. Die Variable Wirtschaftswachstum, die das Vorhandensein und die Wirkung von Wirtschaftskrisen messen soll, deutet, wie vorhergesagt, auf eine konfliktreduzierende Wirkung hin. In Modell 1 verfehlt diese Verbindung nur knapp das Signifikanzniveau. Die Variable übt jedoch in Modell 2 einen klaren Einfluss aus. Es scheint also, dass Wirtschaftskrisen ein Land bedeutend destabilisieren können. Die fehlende Signifikanz des quadrierten Demokratiegrades weist auf eine lineare Form des Verhältnisses zwischen Protesten und Demokratie hin. In Demokratien ist mit mehr Protestverhalten zu rechnen. Diese positive Verbindung lässt sich mit den geringeren Repressionen und freierer Meinungsäußerung in demokratischeren Staaten erklären. Bevölkerung zeigt einen hoch signifikanten, positiven Einfluss auf Instabilität. Das heißt, die großen Länder sind instabiler als die kleinen. Dies steht im Einklang mit der Hypothese, dass große Länder heterogener sind und dass deshalb dort das Konfliktrisiko höher ist.

In den Modellen 3 und 4 ersetzen wir Protest mit politischer Gewalt als Indikator für Instabilität. Hier erweisen sich viele Kontrollvariablen als nicht signifikant. Wie erwartet, ist in Autokratien und Demokratien das Risiko geringer, während es in Regimen, die dazwischen liegen, höher ist. Dies ist zu erkennen am positiven Vorzeichen des Demokratiegrades in einfacher Form und am negativen Vorzeichen des Demokratiegrades in quadrierter Form. Somit findet die These Unterstützung, diese Beziehung weise die Form einer nach unten gekehrten Parabel auf. Beide Variablen haben ein Signifikanz-Niveau von weniger als 1 Prozent. In Modell 4 können wir auch einen positiven Zusammenhang zwischen der Bevölkerungsgröße und Gewalt entdecken. Zwischen Offenheit und politischer Gewalt besteht ein negativer Zusammenhang. Die Wahrscheinlichkeit, dass diese Beziehung durch Zufall zustande kommt, ist geringer als 5 Prozent. Dahingegen scheint der außenwirtschaftliche Öffnungsprozess in Modell 4 keine Auswirkungen auf politische Gewalt zu haben.

Schließlich sehen wir uns Instabilität in Form von Staatsversagen an, also einer abhängigen Variable, die auf den Daten des State Failure-Projekts basiert. Die u-förmige Beziehung zwischen Demokratiegrad und Staatsversagen lässt sich nicht erkennen. Stattdessen sind beide Variablen positiv, der quadrierte Demokratiegrad nicht signifikant. Wir finden mit sehr hoher Wahrscheinlichkeit Unterstützung für die Hypothese, dass große Staaten anfälliger sind für Instabilität. Der Entwicklungsstand hat zwar das erwartete negative Vorzeichen, ist aber nicht statistisch signifikant. Wirtschaftswachstum erweist sich ebenfalls als nicht signifikant. Beide Variablen, Offenheit und Öffnung, haben ein negatives Vorzeichen, sind statistisch aber nicht signifikant. ${ }^{7}$

7 Alternativ zum GEE Ansatz haben wir die zeitliche Abhängigkeit mit einer Methode nach Beck, Katz und Tucker (1998) kontrolliert. Dabei wird jedem Modell eine entsprechende Variable beigefügt, die die Stabilität in der Vergangenheit misst. Diese Variable ist auch theore- 
Zusammenfassend können wir aus den bisherigen Tests schließen, dass eine geöffnete Außenwirtschaft langfristig mit weniger politischer Instabilität in Verbindung steht. Für diesen Zusammenhang finden wir jedoch nur bedingte Unterstützung. Kurzfristig scheint der Öffnungsprozess jedoch keine Auswirkungen auf Instabilität zu haben.

In der nächsten Testreihe untersuchen wir weitere Kontrollvariablen, die in der Literatur identifiziert wurden. Wir wollen sicherstellen, dass unsere Ergebnisse nicht davon beeinflusst werden, dass wir wichtige Faktoren ausgeschlossen haben. Diese Analysen basieren auf den Modellen von politischer Gewalt. Unser Interesse gilt in verstärktem Maße der Klärung der gewalttätigen Form von Instabilität. Eine ungleiche Einkommensverteilung ist eine in der Literatur häufig genannte Ursache für politische Instabilität (Muller/Seligson 1987, Boswell/Dixon 1993, Alesina/Perotti 1996). Zur Messung der Einkommensverteilung verwenden wir den Gini-Koeffizienten, ein Ungleichheitsmaß, das angibt, wie weit eine Verteilung von absoluter Gleichheit entfernt ist mit Daten von Deininger und Squire (1996). Theoretisch ist diese Kontrolle von erheblicher Bedeutung, unseren Ergebnissen zufolge ist sie jedoch statistisch nicht signifikant.

Neben Einkommensungleichheit wird ethnische Fraktionalisierung sehr häufig in Zusammenhang mit politischer Instabilität und politischen Konflikten gebracht. Die Forschung ist sich aber noch darüber uneins, welche Form dieses Verhältnis annimmt. Einerseits nimmt man an, dass die Konfliktgefahr am größten ist, wenn zwei etwa gleich starke Gruppen aufeinander treffen, da jede dieser Gruppen vermutet, aus einem Konflikt siegreich hervorzugehen und auch die Koordinationskosten für die Bildung von Rebellengruppen am niedrigsten sind (Henderson/Singer 2000, Collier/Hoeffler 1998). Auf der anderen Seite wird argumentiert, dass die ethnische Dominanz einer Gruppe am gefährlichsten sei, da die dominierende Gruppe dabei den Anreiz hat, ihre Stärke auszunutzen, um die andere Gruppe ausbeuten zu können (Auvinen 1997; Collier 2001). Wir untersuchen also die Hypothese, dass ethnische Fraktionalisierung das Risiko von innenpolitischem Konflikt erhöht, wozu wir Daten von Collier und Hoeffler (2000) verwenden. Die Variable hat ein negatives Vorzeichen, ist jedoch statistisch nicht signifikant und hat keine Auswirkung auf die anderen Variablen. ${ }^{8}$

Anhand unseres Basismodells von Gewalt haben wir auch untersucht, ob eine Kollinearität von Offenheit oder Öffnung mit einer der Kontrollvariablen die Ergebnisse beeinflusst. So liegt z.B. die Vermutung nahe, dass die Demokratievariablen die Auswirkung von Offenheit beeinträchtigen, da etwa Bliss und Russett (1998) annehmen, dass demokratische Staaten auch in ihren Handelsbeziehungen offener sind. Der Ausschluss von Entwicklungsstand oder Wirtschaftswachstum führt nicht zu veränderten

tisch von Interesse, da sie die historischen Erfahrungen einer Gesellschaft berücksichtigt (Collier/Hoeffler 2001). Errechnet wurde diese Variable mit Binary Time-Series-Cross-Section Data Analysis Utility Version 4.0.4. (Tucker, 1999). Die Ergebnisse, die wir aufgrund dieses Alternativansatzes erzielten, gleichen den hier berichteten Resultaten.

8 An dieser Stelle möchten wir darauf hinweisen, dass unser Fokus auf dem Zusammenhang zwischen außenwirtschaftlicher Liberalisierung und Instabilität liegt und wir mit Hinzunehmen dieser Variable lediglich die Verzerrung reduzieren möchten, die auf den Verzicht auf eine wichtige Variable zurückgeht (omitted variable bias). Wir erheben jedoch nicht den Anspruch, einen Beitrag jenem wichtigen Segment der Konfliktursachenforschung zu leisten, das sich mit möglicherweise ethnisch motivierter Gewalt beschäftigt. 
Ergebnissen von Offenheit oder Öffnung. Wenn wir allerdings die Demokratievariablen aus der Gleichung entfernen, verliert Offenheit an Signifikanz. Die gewaltreduzierende Bedeutung von Offenheit ist also nicht ganz unabhängig vom Regimetyp. Die Einbeziehung einer Interaktionsvariablen zwischen Demokratie und Offenheit erweist sich jedoch als nicht aufschlussreich, da sie insignifikant ist. Wenn Bevölkerung aus der Gleichung genommen wird, erhöht sich das Signifikanz-Niveau von Offenheit enorm $(\mathrm{p}<.001)$. In diesem Fall jedoch kontrolliert Offenheit eher für die Tatsache, dass kleine Länder offener sind. Ein Varianzinflationstest zeigt allerdings, dass Multikollinearität kein Problem in unserem Modell darstellt. ${ }^{9}$

Zum Abschluss werfen wir noch einen kurzen Blick auf die substanzielle Bedeutung der Ergebnisse. Dazu haben wir hypothetische Fälle geschaffen, wobei wir zuerst einen Ausgangsfall geschaffen haben, bei dem für alle Variablen die Durchschnittswerte verwendet werden. Anschließend erhöhen wir nacheinander jeweils eine Variable um eine Standardabweichung. Tabelle 3 fasst diese Simulationen zusammen.

Tabelle 3: Substanzielle Effekte

\begin{tabular}{lccc}
\hline & Protest & Politische Gewalt & Staatsversagen \\
\hline $\begin{array}{l}\text { Alle Variablen als Durchschnittswerte } \\
\begin{array}{l}\text { Offenheit erhöht um eine SA; } \\
\text { andere Variablen als Durchschnitt }\end{array}\end{array}$ & -16.33 & 0.39 & 0.22 \\
$\begin{array}{l}\text { Entwicklung erhöht um eine SA; } \\
\text { andere Variablen als Durchschnitt }\end{array}$ & $+28.8 \%$ & $-15.8 \%$ & $-2.5 \%$ \\
$\begin{array}{l}\text { Wachstum erhöht um eine SA; } \\
\text { andere Variablen als Durchschnitt }\end{array}$ & $-7.0 \%$ & $-1.5 \%$ & $-16.3 \%$ \\
$\begin{array}{l}\text { Demokratie erhöht um eine SA; } \\
\text { andere Variablen als Durchschnitt }\end{array}$ & $+25.5 \%$ & $+12.2 \%$ & $+35.9 \%$ \\
\hline
\end{tabular}

Wäre Offenheit in Modell 2 um eine Standardabweichung größer, reduzierte sich die Wahrscheinlichkeit um 16,6 Prozent, dass es zu einem Protestverhalten käme. Bei dem Modell für Gewalt sind die Auswirkungen etwas stärker. Wäre Offenheit in Modell 3 um eine Standardabweichung höher, verringerte sich die Wahrscheinlichkeit um 15,8 Prozent, dass es im Folgejahr zu politischer Gewalt käme. Für Staatsversagen beträgt diese Reduktion 2,5 Prozent. Diese Ergebnisse bestätigen, dass außenwirtschaftliche Offenheit substanziell bedeutsam ist für die Verringerung von politischer Gewalt und von Massenprotesten.

9 Wir haben durch einen Residualtest unsere Ergebnisse auf Ausreißerfälle untersucht. In den Modellen 2, 4 und 6 hat der Ausschluss der Ausreißerfälle die Ergebnisse nicht beeinflusst. Der Ausschluss der Ausreißerfälle hat in den Modellen 1, 3 und 5 das Signifikanzniveau von Offenheit jeweils angehoben. 


\section{Zusammenfassung}

In dieser Studie haben wir anhand eines Datensatzes von 90 Entwicklungsländern für die Jahre 1978-97 die beiden Fragen untersucht, ob Freihandel das Risiko politischer Instabilität mindert und ob der Prozess der Öffnung es hingegen erhöht. Wir wollten damit Licht auf die lang- und die kurzfristigen politischen Folgen einer freihändlerischen Orientierung der Außenwirtschaftspolitik werfen und so zugleich einen Beitrag zu einem weitgehend unerforschten Aspekt der Globalisierungsdebatte leisten. Unsere Ergebnisse zeigen, dass weder langfristige Offenheit noch der kurzfristige Öffnungsprozess das Protestverhalten zu beeinflussen scheinen. Bezüglich politischer Gewalt hat freier Handel sowohl langfristig als auch, entgegen unseren Erwartungen, kurzfristig eine konfliktreduzierende Wirkung. Wir müssen also die These verwerfen, dass Länder auf dem Weg hin zu einer offenen Außenwirtschaftspolitik anfälliger für Instabilität seien. Die Regressionsergebnisse lassen eher den gegenteiligen Schluss zu. Länder, die tatsächlich viel Handel treiben, scheinen stabiler zu sein. Dies unterstützt die Grundthese, dass weltwirtschaftliche Integration das innenpolitische Konfliktrisiko mindert. Insofern bieten unsere Resultate eine Ergänzung zum liberalen Frieden auf zwischenstaatlicher Ebene. Die Ergebnisse deuten an, dass freier Handel besonders das Potenzial für politische Gewalt eindämmt. Das zweite und ebenso wichtige Ergebnis der Arbeit ist, dass der Öffnungsprozess das Konfliktrisiko im Durchschnitt nicht erhöht. Möglich ist jedoch auch, dass während der Öffnung die Varianz im Stabilitätsniveau größer ist als in Perioden, in denen das Regulierungsniveau unverändert bleibt. Auf diese Möglichkeit hat Reed (2003) jüngst verwiesen, um den Disput zwischen Barbieri/ Schneider (1999) und Oneal/Russett (1999) zum Einfluss der Offenheit auf zwischenstaatliche Konflikte zu klären.

Auch ein hohes Wirtschaftswachstum, hier interpretiert als die Abwesenheit einer ökonomischen Krise, senkt das Risiko von kollektiven Protestverhalten. In Demokratien und großen Ländern lassen sich jedoch wahrscheinlicher Proteste beobachten. Der umgekehrt u-förmige Effekt des Demokratiegrades, nach dem sowohl Demokratien als auch Diktaturen weniger anfällig für politische Gewalt sind, konnte sich bestätigen.

Die Arbeit selbst ist als erster Versuch zu sehen, die Debatte zu den innenpolitischen Auswirkungen der Globalisierung zu versachlichen. Mit der vorliegenden Studie haben wir begonnen, die Brücke zwischen der Literatur zur außenwirtschaftlichen Öffnung auf der einen und der der Bürgerkriegs- und Konfliktforschung auf der anderen Seite zu schlagen. Ein Problem, das die vorliegende Studie mit anderen makroquantitativen Untersuchungen teilt, ist die oft mangelhafte Qualität und Vollständigkeit der verwendeten Indikatoren. Durch eine Vielzahl an fehlenden Werten werden manche Länder komplett aus der Untersuchung ausgeschlossen. Dies beeinträchtigt natürlich die Ergebnisse und macht, da diese fehlenden Werte oft nicht zufällig sind, Verallgemeinerungen schwieriger. Andererseits hat die Analyse auch wie andere jüngere Studien zur Bürgerkriegsforschung gezeigt, dass wohl wichtige Erklärungsvariablen im Modell fehlen. Dies sind vor allem Faktoren, die den genauen Zeitpunkt der Konfliktauslösung beeinflussen. Gezielte Fallstudien, wie wir sie in einem nächsten Forschungsschritt anstreben, werden die hier nur skizzierten Kausalzusammenhänge verdeutlichen. Ein anderer Forschungsschritt wird darin bestehen, internationale Konfliktdeterminan- 
ten mit in die Analyse einzubeziehen. Wir sind aber zuversichtlich, dass auch bei Erweiterungen unseres Forschungsansatzes das zentrale Resultat dieser makroquantitativen Studie erhalten bleibt: Außenwirtschaftliche Öffnung hat in vielen Ländern langfristig jene positive Wirkungen hervorgerufen, die wir hier abschließend als „Friedensdividende“ der Globalisierung bezeichnen möchten.

\section{Literatur}

Adams, Francis/Satya, Dev Gupta/Mengisteab, Kidane, 1999: Globalization and the Dilemmas of the State in the South. London.

Alesina, Alberto/Drazen, Allen, 1991: Why Are Stabilizations Delayed?, in: American Economic Review $81,1170-1188$.

Alesina, Alberto/Perotti, Roberto, 1996: Income Distribution, Political Instability, and Investment, in: European Economic Review 40, 1203-1228.

Alesina, Alberto/Spolare, Enrico/Wacziarg, Romain, 1997: Economic Integration and Political Disintegration, NBER Working Paper Nr. 6163.

Alesina, Alberto/Wacziarg, Romain, 1998: Openness, Country Size and Government, in: Journal of Public Economics 69, 305-321.

Auvinen, Juha, 1997: Political Conflict in Less Developed Countries 1981-89, in: Journal of Peace Research 34, 177-195.

Barbieri, Katherine/Schneider, Gerald, 1999: Globalization and Peace: Assessing New Directions in the Study of Trade and Conflict, in: Journal of Peace Research 3, 387-404.

Bates, Robert H., 1981: Markets and States in Tropical Africa: the Political Basis of Agricultural Policies. Berkeley.

Beck, Nathaniel/Katz, Jonathan N./Tucker, Richard, 1998: Taking Time Seriously in Binary TimeSeries Cross-Section Analysis, in: American Journal of Political Science 42, 1260-1288.

Bliss, H./Russett, Bruce, 1998: Democratic Trading Partners: The Liberal Connection, 1962-1989, in: The Journal of Politics 60, 1126-1147.

Boswell, Terry/Dixon, William J., 1993: Marx's Theory of Rebellion: A Cross-National Analysis of Class Exploitation, Economic Development, and Violent Revolt, in: American Sociological Review 58, 681-702.

Bourguignon, François/Morrisson, Christian, 1990: Income Distribution, Development and Foreign Trade: A Cross Sectional Analysis, in: European Economic Review 34, 1113-1132.

Bueno de Mesquita, Bruce/Morrow, James D./Siverson, Randolph M./Smith, Alastair, 2000: Political Institutions, Political Survival, and Policy Success, in: Bueno de Mesquita, Bruce/Root, Hilton L. (Hrsg.), Governing for Prosperity. New Haven/London 59-84.

Bussmann, Margit/de Soysa, Indra/Oneal, John R., 2002: The Effect of Foreign Investment on Economic Development and Income Inequality. ZEF-Discussion Paper on Development Policy Nr. 53. Bonn.

Bussmann, Margit/Schneider, Gerald, 2003: The „Peace Dividend“ of Globalization: Foreign Economic Liberalization and Internal War. Konferenzbeitrag zur Jahreskonferenz der International Studies Association. Portland.

Clapham, Christopher, 1996: Africa and the International System. The Politics of State Survival. Cambridge.

CNTS Archive, 2001: Cross-National Time-Series Archive, http://www.databanks.sitehosting.net/ www/main.htm

Collier, Paul, 2001: Implications of Ethnic Diversity, in: Economic Policy 1, 29-66.

Collier, Paul, 2000: Doing Well out of War: An Economic Perspective, in: Berdal, Mats/Malone, David M. (Hrsg.), Greed and Grievance: Economic Agendas in Civil Wars. Boulder.

Collier, Paul/Hoeffler, Anke, 1998: On Economic Causes of Civil War, in: Oxford Economic Papers 50, 563-573. 
Collier, Paul/Hoeffler, Anke, 2000: Greed and Grievance in Civil War, unveröffentlichtes Manuskript, Weltbank.

Deininger, Klaus/Squire, Lyn, 1996: A New Data Set Measuring Income Inequality, in: World Bank Economic Review 10, 565-591.

de Soysa, Indra, 2002: Paradise is a Bazaar? Greed, Creed, and Governance in Civil War, 1989-99, in: Journal of Peace Research 39 (4).

Deutsch, Karl Wolfgang u.a., 1957: Political Community and the North Atlantic Area: International Organization in the Light of Historical Experience. Princeton, N.J.

Edwards, Sebastian, 1997: Trade Policy, Growth, and Income Distribution, in: American Economic Review 87, 205-210.

Ellingsen, Tanja, 2000: Colorful Community or Ethnic Witches' Brew? Multiethnicity and Domestic Conflict During and After the Cold War, in: Journal of Conflict Resolution 44, 228-249.

Esty, Daniel C./Goldstone, Jack/Gurr, Ted Robert/Harff, Barbara/Surko, Pamela T./Unger, Alan N./ Chen, Robert S., 1998: The State Failure Project: Early Warning Research for U.S. Foreign Policy Planning, in: Davies, John L./Gurr, Ted Robert (Hrsg.), Preventive Measures. Building Risk Assessment and Crisis Early Warning Systems. Lanham, MD, 27-38.

Fearon, James D./Laitin, David D., 2000: Ethnicity, Insurgency, and Civil War, unveröffentlichtes Manuskript, Stanford University.

Fernandez, Raquel/Rodrik, Dani, 1991: Resistance to Reform: Status Quo Bias in the Presence of Individual-Specific Uncertainty, in: American Economic Review 81, 1146-1155.

Fischer, Ronald D., 2001: The Evolution of Inequality After Trade Liberalization, in: Journal of Development Economics 66, 555-579.

Frey, Bruno S./Eichenberger, Reiner, 1994: The Political Economy of Stabilization Programmes in Developing Countries, in: European Journal of Political Economy 10, 169-190.

Frey, Bruno S./Pommerehne, Werner W./Schneider, Friedrich/Gilbert, Guy, 1984: Consensus and Dissension Among Economists: An Empirical Inquiry, in: American Economic Review 74, 986 994.

Frieden, Jeffry A., 1991: Debt, Development, and Democracy. Modern Political Economy and Latin America, 1965-1985. Princeton, NJ.

Gleditsch, Nils Petter, 1995: Democracy and the Future of European Peace, in: European Journal of International Relations 1, 539-571.

Gleditsch, Nils Petter/Wallensteen, Peter/Eriksson, Mikael/Sollenberg, Margareta/Strand, Havard, 2002: Armed Conflict 1946-2001: A New Dataset, in: Journal of Peace Research 39, 615-637.

Gurr, Ted Robert, 1970: Why Men Rebel. Princeton, NJ.

Gurr, Ted Robert, 1979: Political Protest and Rebellion in the 1960s: The United States in World Perspective, in: Graham, Hugh Davis/Gurr, Ted Robert (Hrsg.), Violence in America: Historical and Comparative Perspectives. Beverly Hills, CA.

Gurr, Ted Robert, 1994: Minorities at Risk. A Global View of Ethnopolitical Conflicts. Washington, D.C.

Hegre, Høvard/Ellingsen, Tanja/Gates, Scott/Gleditsch, Nils Petter, 2001: Toward a Democratic Civil Peace? Democracy, Political Change, and Civil War, 1816-1992, in: American Political Science Review 95, 33-48.

Henderson, Errol A./Singer, David J., 2000: Civil War in the Post-Colonial World, 1946-1992, in: Journal of Peace Research 37, 275-299.

Hiscox, Michael J., 2001: Class versus Industry Cleavages: Inter-Industry Factor Mobility and the Politics of Trade, in: International Organization 55, 1-46.

Hoogvelt, Ankie, 2000: Globalization and the Postcolonial World, in: Held, D./McGrew, A. (Hrsg.), The Global Transformation Reader: An Introduction to the Globalization Debate. Cambridge.

Jagodzinski, Wolfgang, 1983: Ökonomische Entwicklung und politisches Protestverhalten 19201973. Eine kombinierte Quer- und Längsschnittanalyse, in: Politische Vierteljahresschrift, Sonderheft 14, 18-43.

Kant, Immanuel, 1795 [1986]: Zum ewigen Frieden: ein philosophischer Entwurf. Stuttgart.

King, Gary/Zeng, Langche, 2001: Improving Forecasts of State Failure, in: World Politics 53, 623658. 
Kurtenbach, Sabine/Mehler, Andreas, 2002: Regionalwissenschaften und gewaltsame Konflikte, in: Kurtenbach, Sabine/Mehler, Andreas (Hrsg.), Die Vielfalt von Gewaltkonflikten: Analysen aus regionalwissenschaftlicher Perspektive. Hamburg.

Lipset, Seymour Martin, 1959: Some Social Requisites of Democracy: Economic Development and Political Legitimacy, in: American Political Science Review 53, 69-106.

Lipset, Seymour Martin, 1960: Political Man - The Social Bases of Politics. Garden City, NY.

Marshall, Monty G./Jaggers, Keith, 2000: Polity IV Project: Dataset Users Manual, http://www.bsos. umd.edu/cidcm/inscr/polity/index.htm

Martin, Christian W./Plümper, Thomas/Schneider, Gerald, 2001: Economic Openness in Developing Countries: An Empirical Investigation Using CACAO, unveröffentlichtes Manuskript, Universität Konstanz.

Muller, Edward N./Seligson, Mitchell A., 1987: Inequality and Insurgency, in: American Political Science Review 81, 425-452.

Oneal, John R./Russett, Bruce, 1999: Assessing the Liberal Peace with Alternative Specifications: Trade still Reduces Conflict, in: Journal of Peace Research 36, 423-442.

Pawelka, Peter, 1997: Die politische Ökonomie der Außenpolitik im Vorderen Orient, in: Boeckh, Andreas/Pawelka, Peter (Hrsg.), Staat, Markt und Rente in der internationalen Politik. Wiesbaden, 207-231.

Plümper, Thomas, 2001: Weltwirtschaft und Wohlfahrt, unveröffentlichte Habilitationsschrift, Universität Konstanz.

Plïmper, Thomas/Schneider, Gerald, 2000: The Trilemma of the Protectionist Autocrat: Assessing the Link between Democratization, Governmental Divisions and Foreign Economic Liberalization, Paper presented at the APSA conference, October 1-5, 2000.

Pritzl, Rupert/Friedrich Schneider, 1997: Zur Politischen Ökonomie autokratischer politischer Systeme - Ein theoretischer und empirischer Ansatz, Arbeitspapier Nr. 9713, Institut für Volkswirtschaftslehre, Johannes Kepler Universität Linz.

Reed, William, 2003: Information and Economic Interdependence, in: Journal of Conflict Resolution 47, 54-71.

Rodrik, Dani, 1994: The Rush to Free Trade in the Developing World: Why So Late? Why Now? Will It Last?, in: Haggard, Stephan/Webb, Steven B. (Hrsg.), Voting for Reform: Democracy, Political Liberalization, and Economic Adjustment. New York, 61-88.

Rogowski, Ronald, 1989: Commerce and Coalitions. How Trade Affects Domestic Political Alignments. Princeton, NJ.

Rummel, Rudolph J., 1997: Power Kills. Democracy as a Method of Nonviolence. New Brunswick, NJ.

Russett, Bruce, 1993: Grasping the Democratic Peace. Princeton.

Russett, Bruce/Oneal, John R., 2001: Triangulating Peace: Democracy, Interdependence, and International Organizations. Princeton, NJ.

Sachs, Jeffrey D./Warner, Andrew, 1995: Economic Reform and the Process of Global Integration, in: Brookings Papers on Economic Activity 1, 1-118.

Schlichte, Klaus, 1996: Krieg und Vergesellschaftung in Afrika: Ein Beitrag zur Theorie des Krieges. Münster.

Schneider, Gerald/Barbieri, Katherine/Gleditsch, Nils Petter, 2003: Globalization and Armed Conflict. Lanham, MD.

Schock, Kurt, 1996: A Conjunctural Model of Political Conflict. The Impact of Political Opportunities on the Relationship Between Economic Inequality and Violent Political Conflict, in: Journal of Conflict Resolution 40, 98-134.

Senghaas, Dieter, 1988: Konfliktformationen im internationalen System. Frankfurt/Main.

Tarrow, Sidney, 1989: Struggle, Politics, and Reform: Collective Action, Social Movements, and Cycles of Protest. Ithaca, NY.

Tarrow, Sidney, 1998: Power in Movement. Social Movements and Contentious Politics. 2. Ausg., New York.

Tilly, C., 1978: From Mobilization To Revolution. New York. 
Tucker, Richard, 1999: BTSCS: A Binary Time-Series Cross-Section Data Analysis Utility. Version 4.0.4. Cambridge, MA: Harvard University. http://www.fas.harvard.edu/ $/$ rtucker/programs/ btscs/btscs.html

Tullock, Gordon, 1980: Rent Seeking as a Negative-Sum Game, in: Buchanan, James M./Tollison, Robert D./Tullock, Gordon (Hrsg.), Toward a Theory of the Rent Seeking Society, College Station, $16-36$.

Weede, Erich, 1990: Wirtschaft, Staat und Gesellschaft. Tübingen.

Wood, Adrian, 1994: North-South Trade, Employment and Inequality: Changing Fortunes in a Skill-Driven World. Oxford.

World Bank, 1999: World Development Indicators 1999, CD-ROM. Washington, DC.

Zanger, Sabine, 2000: A Global Analysis of the Effect of Political Regime Changes on Life Integrity Violations, 1977-93, in: Journal of Peace Research 37, 213-233.

Zorn, Christopher J.W., 2001: Generalized Estimating Equation Models for Correlated Data: A Review with Applications, in: American Journal of Political Science 45, 470-490. 